\title{
TITLE:
}

\section{Complex Ginzburg-Landau equation with nonlocal coupling}

$\operatorname{AUTHOR}(S)$ :

Tanaka, D; Kuramoto, Y

\section{CITATION:}

Tanaka, D ...[et al]. Complex Ginzburg-Landau equation with nonlocal coupling. Physical Review E 2003, 68(2): 026219.

ISSUE DATE:

2003-08

URL:

http://hdl.handle.net/2433/49991

RIGHT:

Copyright 2003 American Physical Society 
PHYSICAL REVIEW E 68, 026219 (2003)

\title{
Complex Ginzburg-Landau equation with nonlocal coupling
}

\author{
Dan Tanaka and Yoshiki Kuramoto \\ Department of Physics, Graduate School of Sciences, Kyoto University, Kyoto 606-8502, Japan
}

(Received 17 April 2003; published 26 August 2003)

\begin{abstract}
A Ginzburg-Landau-type equation with nonlocal coupling is derived systematically as a reduced form of a universal class of reaction-diffusion systems near the Hopf bifurcation point and in the presence of another small parameter. The reaction-diffusion systems to be reduced are such that the chemical components constituting local oscillators are nondiffusive or hardly diffusive, so that the oscillators are almost uncoupled, while there is an extra diffusive component which introduces effective nonlocal coupling over the oscillators. Linear stability analysis of the reduced equation about the uniform oscillation is also carried out. This revealed that new types of instability which can never arise in the ordinary complex Ginzburg-Landau equation are possible, and their physical implication is briefly discussed.
\end{abstract}

DOI: 10.1103/PhysRevE.68.026219

PACS number(s): 05.45. $-\mathrm{a}, 47.54 .+\mathrm{r}, 82.40 .-\mathrm{g}$

\section{INTRODUCTION}

Oscillatory reaction-diffusion systems are generally reduced to the complex Ginzburg-Landau (CGL) equation by means of the so-called center-manifold reduction when the local oscillators are close to their supercritical Hopf bifurcation point [1]. This fact led to a now widely accepted view that, without resorting to individual systems, one may concentrate on CGL if one wishes to gain a qualitative understanding of some universal dynamical features shared commonly by a broad class of oscillatory reaction-diffusion systems. This view in fact underlies a vast amount of work in the past (see Refs. [2,3] for review) devoted to CGL. However, due to its local nature of coupling, CGL may fail to capture some important aspects of the dynamics characteristic to a certain class of oscillatory reaction-diffusion systems.

In fact, it was argued recently that a situation may arise where the oscillator coupling becomes effectively nonlocal, and as a consequence the system exhibits such peculiar dynamics as can never be seen in CGL $[4,5]$. This suggests that there remains a yet unexplored area of reaction-diffusion systems where nonlocal effects on the pattern dynamics must seriously be considered. In the present paper, we are concerned with how this area is still accessible within the framework of the center-manifold reduction. It will turn out that this can actually be achieved by a slight extension of the conventional reduction scheme.

Effective nonlocality in coupling may become relevant when the reaction-diffusion system involves three or more chemical components. Suppose that the system of concern is such that the chemical components constituting the local oscillators are free of diffusion, i.e., the oscillators remain uncoupled, while the system involves an extra diffusive component which, for its diffusive nature, plays the role of a coupling agent. By eliminating mathematically this diffusive component, the system becomes a field of nonlocally coupled oscillators possibly involving delay also. The main goal of the present paper is to achieve a reduction of such reaction-diffusion systems to a universal equation of the Ginzburg-Landau type without missing the effects of nonlocality.

It may seem that the center-manifold idea would not work for our purpose because CGL is believed to be quite a general result of the center-manifold reduction. Still there seems to be a way out within the same framework if we slightly extend the conventional method of reduction. Note first that under usual conditions the disappearance of the effects of nonlocality near the bifurcation point comes from that the characteristic wavelength $l_{p}$ of the pattern becomes far longer than the effective coupling radius. If so, it may happen that nonlocality persists even close to the bifurcation point when the system involves a certain parameter whose suitable tuning, as the bifurcation point is approached, keeps $l_{p}$ comparable with the effective radius of coupling. Similar physical idea lies behind the multiple bifurcation theory which aims to capture such complex dynamics as is absent in the vicinity of a simple bifurcation point.

Section II starts with introducing a universal class of oscillatory reaction-diffusion systems involving three or more chemical components. After briefly discussing its physical relevance, we proceed to its reduction near the Hopf bifurcation. If a certain parameter associated with the strength of effective nonlocal coupling is as small as the bifurcation parameter, the reduced equation turns out to take the form of a nonlocally coupled complex Ginzburg-Landau equation. Analytic formulas for some coefficients of this equation are given, which would be of great help in inferring possible ranges of the parameters in the original system where nonlocality-dominated pattern dynamics is expected. Regarding the derivation of the reduced equation, our primary concern is the case when direct oscillator coupling is absent, but the effects of weak diffusive (i.e., direct) coupling will also be considered. In Sec. III, linear stability analysis of our nonlocal CGL is carried out about the uniform oscillation. The resulting eigenvalue spectra, especially those of the phase branch, can be qualitatively different from those of the standard CGL, whose physical implication is discussed. A short summary will be given in the final section.

\section{A UNIVERSAL CLASS OF REACTION-DIFFUSION SYSTEMS AND THEIR REDUCTION}

The reaction-diffusion model of our concern was previously proposed by one of the present authors [4] and is given by the general form 


$$
\begin{gathered}
\partial_{t} \boldsymbol{X}=\boldsymbol{f}(\boldsymbol{X})+k \boldsymbol{g}(S), \\
\tau \partial_{t} S=-S+D \nabla^{2} S+h(\boldsymbol{X}) .
\end{gathered}
$$

Here the $n$-dimensional real vector field $\boldsymbol{f}$ represents a local limit-cycle oscillator with dynamical variable $\boldsymbol{X}$, so that the first equation with $k=0$ represents a field of continuously distributed oscillators without mutual coupling. The full system involves an additional chemical component with concentration $S$ whose dynamics is governed by the second equation. This component simply diffuses and decays at a constant rate, while it is produced locally as represented by the term $h(\boldsymbol{X})$, the production rate depending on the local value of $\boldsymbol{X}$. The dynamics of the local oscillators is influenced in return by the local concentration of $S$, and this effect is represented by the term $\mathrm{kg}(S)$. We do not know any experimental system which can directly be related to our reaction-diffusion model, although biological populations such as cellular slime molds and oscillating yeast cells under glycolysis, and also the recent series experiments by Vanag and Epstein [6] using the Belousov-Zhabotinsky reaction dispersed in water-in-oil aerosol OT microemulsion show a partial similarity to our model in that the coupling between the local oscillators is mediated by some diffusive chemicals.

For the sake of convenience, we inserted in Eq. (2) parameter $\tau$ to indicate explicitly the time constant of $S$, anticipating a limiting case in which $\tau$ is vanishingly small. Note that there is no direct coupling among the local oscillators, while their indirect coupling is provided by the diffusive chemical represented by $S$. In later discussions, we will generalize the above model by assuming $\boldsymbol{g}$ and $h$ to depend both on $\boldsymbol{X}$ and $S$, and also by including a small diffusion term, i.e., weak direct coupling in Eq. (1). Throughout the present paper, the spatial extension of the system is supposed sufficiently large.

Let the spatially uniform steady state of our system be given by $(\boldsymbol{X}, S)=(0,0)$, or equivalently, we measure $\boldsymbol{X}$ and $S$ always from their equilibrium values. Thus, we clearly have $h(0)=0$. It is also convenient to arrange $\boldsymbol{f}$ and $\boldsymbol{g}$ so that the equalities $\boldsymbol{f}(0)=\boldsymbol{g}(0)=0$ may be satisfied. If we like, $S$ may be eliminated from the system by solving Eq. (2), which can be done explicitly because the equation is linear. If the spatial dimension is $d$, the solution of Eq. (2) is given by

$$
\begin{aligned}
S(\boldsymbol{r}, t)= & (2 \pi)^{-d} \int d \boldsymbol{q} \exp (i \boldsymbol{q} \cdot \boldsymbol{r}) \int_{-\infty}^{t} \frac{d t^{\prime}}{\tau} \\
& \times \exp \left(-\left(1+D q^{2}\right) \frac{t-t^{\prime}}{\tau}\right) h_{\boldsymbol{q}}\left(t^{\prime}\right),
\end{aligned}
$$

where $h_{\boldsymbol{q}}\left(t^{\prime}\right)$ is the spatial Fourier transform of $h\left(\boldsymbol{X}\left(\boldsymbol{r}, t^{\prime}\right)\right)$. Equation (1) with $S$ given by Eq. (3) constitutes a field of nonlocally coupled oscillators. Note that the nonlocality appears in time as well as in space, and the characteristic scales in time and space associated with the nonlocality are given by $\tau$ and $D^{1 / 2}$, respectively. This fact will be used in the discussion below.

Suppose that $\boldsymbol{f}$ involves a parameter $\mu$ such that if $\mu<0$ each local system given by Eqs. (1) and (2) with $D=0$ has a stable fixed point $(\boldsymbol{X}, S)=(0,0)$, while this becomes oscillatory unstable for $\mu \geqslant 0$; namely, at $\mu=0$ a pair of complex conjugate eigenvalues of the Jacobian associated with each local system about the fixed point cross the imaginary axis of the complex plane, while the other eigenvalues all remain in the left half plane. It is a well-known fact that the centermanifold reduction can be applied to reaction-diffusion systems near the Hopf bifurcation point of the local oscillators [1]. This leads generally to the CGL equation

$$
\partial_{t} A=\mu \sigma A-\beta|A|^{2} A+\alpha \nabla^{2} A,
$$

where the amplitude $A$ and the parameters $\sigma, \alpha$, and $\beta$ are generally complex. The small parameter $\mu$ has been retained in Eq. (4) so that $A$, $t$, and $\boldsymbol{r}$ scale like $|\mu|^{1 / 2},|\mu|^{-3 / 2}$, and $|\mu|^{-1 / 2}$, respectively.

It is clear that the above reduced form of reactiondiffusion systems remains valid also for our particular system given by Eqs. (1) and (2). Since CGL is a diffusively coupled (i.e., locally coupled) system, it may seem that effective nonlocality in coupling characteristic to our system disappears completely near the bifurcation point. The reason for the disappearance of nonlocality is clear. This is because the characteristic wavelength $l_{p}$ of the field $\boldsymbol{X}$ becomes longer and longer as the bifurcation point is approached like $l_{p} \sim|\mu|^{-1 / 2}$ so that the effective coupling radius given by $D^{1 / 2}$ comes to fall well within this scale, which gives nothing but the definition of local coupling. In what follows, we will be concerned with a special situation in which spatial (and possibly temporal) nonlocality can survive even close to the bifurcation point, so that the reduced equation involves nonlocal coupling rather than diffusive coupling. The same result was used already in earlier works [4,5] for the particular case of vanishingly small $\tau$ without showing how the reduction can actually be achieved. We will develop below the reduction procedure explicitly including the case of finite $\tau$.

Consider our system given by the form of Eqs. (1) and (3) for which Eq. (4) gives the right reduced form near $\mu=0$ provided there is no small parameter other than $\mu$. It is clear that the diffusion term in Eq. (4) is the reduced form of the coupling term $k \boldsymbol{g}(S)$ in Eq. (1). This implies that $|\alpha|$ $=O(|k|)$. Disappearance of spatial nonlocality is consistent with the fact that the characteristic wavelength $l_{p}$ estimated from the dimensional argument for Eq. (4), which confirms $l_{p}=O\left(|\mu / k|^{-1 / 2}\right)$, is far larger than the effective coupling radius given by $D^{1 / 2}$ provided $k$ remains an ordinary magnitude. This consistency is apparently broken if $k$ becomes as small as $\mu$ by which $l_{p}$ becomes independent of $\mu$ and hence can be comparable with the coupling radius. Putting it differently, spatial nonlocality should remain relevant near the bifurcation point provided the strength of coupling between the local oscillators and the diffusive component becomes so weak as to satisfy

$$
k \sim O(|\mu|) .
$$

Thus, what we do next is to find a reduced equation valid near the doubly singular point $(\mu, k)=(0,0)$. Before proceeding to this issue, however, we make a remark on temporal nonlocality. Temporal nonlocality, which is characterized by the time constant $\tau$, may be relevant to the dynamics even 
near the bifurcation point. However, this effect would never appear as a non-Markoffian form of the reduced equation for the amplitude $A$ because the time scale associated with the variation of $A$ is much longer than $\tau$. Still, $\tau$ could be comparable with the period of the basic oscillation. Then, as we see below, the effect of delay may appear in the reduced equation through the change in the space dependence of the coupling function. Specifically, when $\tau$ is finite, the decay of the coupling function with distance becomes oscillatory rather than monotone.

Suppose first that $k=0$. Then the reduced form of the ordinary equation (1) is given by Eq. (4) with $\alpha=0$. Although its derivation is routine, we now recapitulate it for the purpose of explaining later how easily the reduced equation can be generalized when a small coupling term is introduced.

Let the Taylor expansion of $\boldsymbol{f}(\boldsymbol{X})$ in terms of $\boldsymbol{X}$ be written as

$$
\boldsymbol{f}(\boldsymbol{X})=L(\boldsymbol{X})+M(\boldsymbol{X} X, \boldsymbol{X})+N(\boldsymbol{X}, \boldsymbol{X}, \boldsymbol{X}+\cdots,
$$

where the vector functions $\boldsymbol{L}(\boldsymbol{x}), \boldsymbol{M}(\boldsymbol{x}, \boldsymbol{y}), \boldsymbol{N}(\boldsymbol{x}, \boldsymbol{y}, \boldsymbol{z}), \cdots$ are linear functions of each argument and also symmetric in these vectors. In what follows, $\boldsymbol{L}(\boldsymbol{x})$ will also be expressed as $\boldsymbol{L} \boldsymbol{x}$ in terms of the Jacobian $L$ of $\boldsymbol{f}$ at $\boldsymbol{X}=0$. Regarding the $\mu$ dependence of $\boldsymbol{L}$ ( or $\boldsymbol{L}$ ), $\boldsymbol{M}, \boldsymbol{N}, \cdots$ appearing in the above expansion, we need to consider it only for the Jacobian $L$ to the first order in $\mu$ such as $L=L_{0}+\mu L_{1}$; higher order corrections to $L$ as well as $\mu$ dependence of $\boldsymbol{M}, \boldsymbol{N}$, etc. are irrelevant to the reduced equation to the leading order. Let the pure imaginary eigenvalues at $\mu=0$ be $\pm i \omega_{0}$, and the corresponding right eigenvectors and its complex conjugate are written as $\boldsymbol{U}$ and $\overline{\boldsymbol{U}}$, respectively. The corresponding left eigenvectors and its complex conjugate are denoted by $\boldsymbol{U}^{*}$ and $\overline{\boldsymbol{U}}^{*}$, respectively. These eigenvectors satisfy $\boldsymbol{U}^{*} \cdot \overline{\boldsymbol{U}}$ $=\overline{\boldsymbol{U}}^{*} \cdot \boldsymbol{U}=0$ and $\boldsymbol{U}^{*} \cdot \boldsymbol{U}=\overline{\boldsymbol{U}}^{*} \cdot \overline{\boldsymbol{U}}=1$. If $\mu$ is nonvanishing but small, the eigenvalues change to $\pm i \omega_{0}+\mu \lambda_{ \pm}$, where $\bar{\lambda}_{+}=\lambda_{-}$.

To the lowest order in $\mu$, the original field $\boldsymbol{X}$ and the complex amplitude $A$ are mutually related via

$$
\boldsymbol{X}(t)=e^{i \omega_{0} t} \boldsymbol{U} A(t)+\text { c.c.. }
$$

Thus, in this approximation we have $\partial_{t} A$ $=\exp \left(-i \omega_{0} t\right) \boldsymbol{U}^{*} \cdot\left(\dot{\boldsymbol{X}}-L_{0} \boldsymbol{X}\right)$. This means further that the right-hand side of Eq. (4) with $\alpha=0$ is identical with the reduced form of $\exp \left(-i \omega_{0} t\right) \boldsymbol{U}^{*} \cdot\left[\left(\boldsymbol{f}(\boldsymbol{X})-L_{0} \boldsymbol{X}\right)\right]$ or

$$
\begin{aligned}
\mu \sigma A-\beta|A|^{2} A \simeq & e^{-i \omega_{0} t} \boldsymbol{U}^{*} \cdot\left[\boldsymbol{f}(\boldsymbol{X})-L_{0} \boldsymbol{X}\right] \\
= & e^{-i \omega_{0} t} \boldsymbol{U}^{*} \cdot\left[\mu L_{1} \boldsymbol{X}+\boldsymbol{M}(\boldsymbol{X}, \boldsymbol{X})\right. \\
& +\boldsymbol{N}(\boldsymbol{X}, \boldsymbol{X}, \boldsymbol{X}+\cdots] .
\end{aligned}
$$

The standard analysis determines the coefficients $\sigma$ and $\beta$ in terms of some parameters of the equations before reduction. It is clear that the linear coefficient $\sigma$ is given by

$$
\sigma=\boldsymbol{U}^{*} \cdot L_{1} \boldsymbol{U}=\lambda_{+} .
$$

For obtaining $\beta$, the lowest order expression for $\boldsymbol{X}$ given by Eq. (7) is insufficient, and we have to use a more precise formula including the next order term:

$$
\begin{aligned}
\boldsymbol{X}(t)= & e^{i \omega_{0} t} \boldsymbol{U} A(t)+\text { c.c. }+e^{2 i \omega_{0} t} \boldsymbol{V}_{+} A^{2}+e^{-2 i \omega_{0} t} \boldsymbol{V}_{-} \bar{A}^{2} \\
& +\boldsymbol{V}_{0}|A|^{2},
\end{aligned}
$$

where

$$
\begin{gathered}
\boldsymbol{V}_{+}=\overline{\boldsymbol{V}}_{-}=-\left(L_{0}-2 i \omega_{0}\right)^{-1}(\boldsymbol{M}, \boldsymbol{U}, \boldsymbol{U}), \\
\boldsymbol{V}_{0}=-2 L_{0}^{-1}(\boldsymbol{M}, \boldsymbol{U}, \overline{\boldsymbol{U}}) .
\end{gathered}
$$

Using these quantities, $\beta$ is given by

$\beta=-2 \boldsymbol{U}^{*} \cdot M\left(\boldsymbol{U}, \boldsymbol{V}_{0}\right)-2 \boldsymbol{U}^{*} \cdot \boldsymbol{M}\left(\overline{\boldsymbol{U}}, \boldsymbol{V}_{+}\right)-3 \boldsymbol{U}^{*} \cdot \boldsymbol{N}(\boldsymbol{U}, \boldsymbol{U}, \overline{\boldsymbol{U}})$.

Suppose that the vector field $f$ is modified slightly to $f$ $+\boldsymbol{p}$. It is clear that the corresponding reduced equation must also be modified slightly with an additive term $\exp \left(-i \omega_{0} t\right) \boldsymbol{U}^{*} \cdot \boldsymbol{p}$. The specific form of $\boldsymbol{p}$ of our concern is the small coupling term $k \boldsymbol{g}(S)$ in Eq. (1) with $S$ given by Eq. (3). The original variable $X$ and the reduced one $A$ must now be regarded as depending on space as well as on time. Thus, our problem is to find a reduced form of the quantity

$$
k \exp \left(-i \omega_{0} t\right) \boldsymbol{U}^{*} \cdot \boldsymbol{g}(S) \equiv k \tilde{\boldsymbol{p}}
$$

using Eq. (3). Since $k$ is already small, we only need to consider the most dominant contribution to $\tilde{\boldsymbol{p}}$. Noting that $\boldsymbol{g}(0)=0$, we may use a linear approximation

$$
\begin{aligned}
\boldsymbol{g}(S) \simeq \boldsymbol{g}_{0} S \simeq & \frac{\boldsymbol{g}_{0}}{(2 \pi)^{d}} \int d \boldsymbol{q} \exp (i \boldsymbol{q} \cdot \boldsymbol{r}) \int_{-\infty}^{t} \frac{d t^{\prime}}{\tau} \\
& \times \exp \left(-\left(1+D q^{2}\right) \frac{t-t^{\prime}}{\tau}\right) \boldsymbol{h}_{0} \cdot \boldsymbol{X}_{\boldsymbol{q}}\left(t^{\prime}\right),
\end{aligned}
$$

where $\boldsymbol{g}_{0}=d \boldsymbol{g} /\left.d S\right|_{S=0}$ and $\boldsymbol{h}_{0}=d h(\boldsymbol{X}) /\left.d \boldsymbol{X}\right|_{\boldsymbol{X}=0}$. Thus, using Eq. (7) with $\boldsymbol{X}$ and $A$ supposed to depend also on $\boldsymbol{r}$, we have

$$
\begin{aligned}
\tilde{\boldsymbol{p}}= & \frac{\eta}{(2 \pi)^{d}} \int d \boldsymbol{q} \exp (i \boldsymbol{q} \cdot \boldsymbol{r}) \int_{-\infty}^{t} \frac{d t^{\prime}}{\tau} \\
& \times \exp \left(-\left(1+D q^{2}\right) \frac{t-t^{\prime}}{\tau}-i \omega_{0}\left(t-t^{\prime}\right)\right) A_{\boldsymbol{q}}\left(t^{\prime}\right),
\end{aligned}
$$

where

$$
\eta=\left(\boldsymbol{U}^{*} \cdot \boldsymbol{g}_{0}\right)\left(\boldsymbol{h}_{0} \cdot \boldsymbol{U}\right)
$$

Note that Eq. (16) ignores the contribution from the complex conjugate of $A(t)$ which would give rise to a rapidly oscillating component of $\tilde{\boldsymbol{p}}$. This is allowed because such a component would be averaged out in the equation describing the 
slow evolution of $A(t)$. Since the time integral in Eq. (16) extends practically over the interval between $t-\tau$ and $t$, the slowly varying amplitude $A_{q}\left(t^{\prime}\right)$ may safely be replaced with $A_{q}(t)$. In this way, we obtain

$$
k \tilde{\boldsymbol{p}}=k \eta^{\prime} \int d \boldsymbol{r}^{\prime} G\left(\boldsymbol{r}-\boldsymbol{r}^{\prime}\right) A\left(\boldsymbol{r}^{\prime}, t\right),
$$

where

$$
G(\boldsymbol{r})=\frac{1}{(2 \pi)^{d}} \int d \boldsymbol{q} e^{i \boldsymbol{q} \cdot \boldsymbol{r}} \frac{1+i \omega_{0} \tau}{D q^{2}+1+i \omega_{0} \tau}
$$

and

$$
\eta^{\prime}=\frac{\eta}{1+i \omega_{0} \tau} .
$$

Note that $G(\boldsymbol{r})$ satisfies the normalization condition

$$
\int d \boldsymbol{r} G(\boldsymbol{r})=1
$$

Thus, the final form of the reduced equation becomes

$$
\partial_{t} A=\mu \sigma A-\beta|A|^{2} A+k \eta^{\prime} \int d \boldsymbol{r}^{\prime} G\left(\boldsymbol{r}-\boldsymbol{r}^{\prime}\right) A\left(\boldsymbol{r}^{\prime}, t\right),
$$

which we call nonlocally coupled complex Ginzburg-Landau equation or simply nonlocal CGL. It is clear that the situation of interest is such that $k=O(|\mu|)$ for which the coupling term in the reduced equation is balanced in magnitude with the other terms even if the characteristic wavelength is independent of $\mu$. We assume that the bifurcation is supercritical, i.e., the real part of $\beta$ is positive.

A few generalizations of the original system [Eqs. (1) and (2)] can be made. First, $\boldsymbol{g}$ and $h$ may depend both on $\boldsymbol{X}$ and $S$. Since the most dominant part of these quantities alone is relevant to the reduced equation, one may safely approximate $\boldsymbol{g}(\boldsymbol{X}, S)$ and $h(\boldsymbol{X}, S)$ as $\boldsymbol{g}(\boldsymbol{X}, S)=\boldsymbol{g}(\boldsymbol{X}, 0)+\boldsymbol{g}(0, S)$ and $h(\boldsymbol{X}, S)=h(\boldsymbol{X}, 0)+h(0, S)$, respectively. The resulting new term $k \boldsymbol{g}(\boldsymbol{X}, 0)$ may slightly modify $\boldsymbol{f}(\boldsymbol{X})$, but the modified $\boldsymbol{f}(\boldsymbol{X})$ may again be denoted by $\boldsymbol{f}(\boldsymbol{X})$. Furthermore, because $S$ is small, $h(0, S)$ is practically linear in $S$. Thus, this quantity simply modifies the linear decay rate of $S$ which can be normalized by a suitable rescaling of time. In this way, the final result of reduction is unchanged except that $\boldsymbol{g}(S)$ and $h(\boldsymbol{X})$ are replaced with $\boldsymbol{g}(0, S)$ and $h(\boldsymbol{X}, 0)$, respectively. As the second generalization, we may include in Eq. (1) a diffusion term such as

$$
\partial_{t} \boldsymbol{X}=\boldsymbol{f}(\boldsymbol{X})+\hat{\delta} \nabla^{2} \boldsymbol{X}+\boldsymbol{k} \boldsymbol{g}(S),
$$

where $\hat{\delta}$ is a diagonal diffusion matrix with non-negative elements. In parallel with the above argument for obtaining a reduced form of the nonlocal coupling term, the reduced form of the quantity $\exp \left(-i \omega_{0} t\right) \boldsymbol{U}^{*} \cdot \hat{\delta} \nabla^{2} \boldsymbol{X}$ will then be added to the right-hand side of Eq. (22). To the lowest order approximation, one may apply Eq. (7) for $\boldsymbol{X}$, by which the above quantity becomes $\delta \nabla^{2} A$ where $\delta$ is a complex number with positive real part, and is given by

$$
\delta=\boldsymbol{U}^{*} \cdot \hat{\delta} \boldsymbol{U} .
$$

Thus, Eq. (22) is modified as

$$
\partial_{t} A=\mu \sigma A-\beta|A|^{2} A+\delta \nabla^{2} A+k \eta^{\prime} \int d \boldsymbol{r}^{\prime} G\left(\boldsymbol{r}-\boldsymbol{r}^{\prime}\right) A\left(\boldsymbol{r}^{\prime}, t\right) .
$$

In the conventional reduction of reaction-diffusion systems, $|\delta|$ is assumed to be of ordinary magnitude, so that the diffusion term can be balanced with the linear and cubic terms in magnitude only if the characteristic wavelength of $A$ scales like $|\mu|^{-1 / 2}$. However, the last property of $A$ contradicts the particular situation of our concern in which the characteristic wavelength remains independent of $\mu$. Therefore, in what follows, we assume that $|\delta|$ as well as $k$ is of the order of $\mu$, by which all terms on the right-hand side of Eq. (25) are balanced with each other, and the coupling nonlocality represented by the last term can survive.

It is more convenient to write Eq. (25) in the form

$$
\begin{aligned}
\partial_{t} A= & \mu \sigma^{\prime} A-\beta|A|^{2} A+\delta \nabla^{2} A \\
& +k \eta^{\prime} \int d \boldsymbol{r}^{\prime} G\left(\boldsymbol{r}-\boldsymbol{r}^{\prime}\right)\left[A\left(\boldsymbol{r}^{\prime}, t\right)-A(\boldsymbol{r}, t)\right],
\end{aligned}
$$

where $\sigma^{\prime}=\sigma+\mu^{-1} k \eta^{\prime}$. With this form, the coupling term can be approximated by a diffusion term when the characteristic wavelength of $A(\boldsymbol{r}, t)$ is sufficiently longer than the coupling radius. Note that $\sigma^{\prime}$ remains of ordinary magnitude because $k=O(\mu)$ by assumption. Hereafter, we assume that the system is supercritical or

$$
\operatorname{Re} \sigma^{\prime}>0 .
$$

An additional remark should be made on the functional form of the coupling function $G$ in connection with the time scale $\tau$ of the diffusive component $S$. As implied by Eq. (16), finite $\tau$ generally gives rise to memory effects or temporal nonlocality after the variable $S$ has been eliminated. However, as was noted before, the reduced equation is free from memory effects because the time scale of the slowly varying amplitude $A$ is much longer than $\tau$. We also noted that because $\tau$ may be comparable with the period $2 \pi / \omega_{0}$ of the fundamental oscillation, the effect of delay in coupling should be relevant to the reduced dynamics. Actually, as is clear from Eq. (19), the effect of finite $\tau$ appears in the coupling function $G$. For one-dimensional systems, in particular, the coupling function is simply expressed as

$$
G(x)=\frac{1}{2}\left(\alpha_{+}+i \alpha_{-}\right) e^{-\left(\alpha_{+}+i \alpha_{-}\right)|x|},
$$

where

$$
\alpha_{ \pm}=\left(\frac{ \pm 1+\sqrt{1+\theta^{2}}}{2 D}\right)^{1 / 2},
$$


and $\theta=\omega_{0} \tau$. If $\tau$ is vanishing, $G(x)$ decays exponentially with $|x|$, the decay length being given by $D^{1 / 2}$, whereas the decay becomes oscillatory when $\tau$ is finite. Thus, the effect of delay in coupling is such that the complex amplitude $A\left(x^{\prime}, t\right)$ in the coupling term is multiplied by a factor of $\exp \left(-i \alpha_{-}\left|x-x^{\prime}\right|\right)$, or equivalently, the phase of $A\left(x^{\prime}, t\right)$, denoted by $\phi\left(x^{\prime}, t\right)$, is replaced with $\phi\left(x^{\prime}, t\right)-\alpha_{-}\left|x-x^{\prime}\right|$. In the physical language, this means that the phase at the spatial point $x^{\prime}$ experienced by the oscillator at $x$ through the delayed coupling cannot be its current value $\phi\left(x^{\prime}, t\right)$ but should be the value at some time $t-t_{0}$ in the past, because the phase information travels at a finite speed. If this speed is constant and the oscillation at $x^{\prime}$ is nearly regular, then we have $\phi\left(x^{\prime}, t-t_{0}\right)$ equal to $\phi\left(x^{\prime}, t\right)$ plus something proportional to the distance $\left|x-x^{\prime}\right|$, justifying the above result.

Coming back to general space dimensions, Eq. (26) with the coupling function given by Eq. (19) involves many parameters. However, some of the parameters can be eliminated by suitable transformations of some variables. First, the imaginary part of the linear coefficient $\mu \sigma^{\prime}$ vanishes through the transformation $A \rightarrow A \exp \left[i \mu \operatorname{Im} \sigma^{\prime} t\right]$. Second, one may rescale $A, t$, and $r$ in such a way that $\operatorname{Re} \beta, \mu \operatorname{Re} \sigma^{\prime}$, and $D$ may all become unity. In this way, we may write Eq. (26) as

$$
\begin{aligned}
\partial_{t} A= & A-\left(1+i c_{2}\right)|A|^{2} A+\left(\delta_{1}+i \delta_{2}\right) \nabla^{2} A \\
& +K\left(1+i c_{1}\right) \int d \boldsymbol{r}^{\prime} G\left(\boldsymbol{r}-\boldsymbol{r}^{\prime}\right)\left[A\left(\boldsymbol{r}^{\prime}, t\right)-A(\boldsymbol{r}, t)\right],
\end{aligned}
$$

where the coupling function $G$ is defined as an integral form given by Eq. (19), or

$$
\begin{gathered}
G(\boldsymbol{r})=\frac{1}{(2 \pi)^{d}} \int d \boldsymbol{q} e^{i \boldsymbol{q} \cdot \boldsymbol{r}} G_{q}, \\
G_{q} \equiv \frac{1+i \theta}{q^{2}+1+i \theta} .
\end{gathered}
$$

The reduced equation now involves six independent parameters $c_{1}, c_{2}, K, \theta, \delta_{1}$, and $\delta_{2}$ all of which are independent of smallness parameters $\mu, k$, and $\delta$. Note that the coupling coefficient in Eq. (30) is related to some original parameters through

$$
K\left(1+i c_{1}\right)=\frac{k \eta^{\prime}}{\mu \operatorname{Re} \sigma^{\prime}}=\frac{k \eta^{\prime}}{\mu \operatorname{Re} \sigma+k \operatorname{Re} \eta^{\prime}}
$$

or

$$
K=1-\frac{\operatorname{Re} \sigma}{\operatorname{Re} \sigma^{\prime}}
$$

so that, by combining the last equation with the inequality (27) and the original assumption $\operatorname{Re} \sigma>0$, we have a restrictive condition

$$
K<1 \text {. }
$$

\section{EIGENVALUE SPECTRUM ABOUT THE UNIFORM OSCILLATION}

It is clear that Eq. (30) admits a family of plane wave solutions $A_{k}(\boldsymbol{r}, t)=R \exp [i(\boldsymbol{k} \cdot \boldsymbol{r}-\Omega t)]$, the stability of which is extremely important to the understanding of the pattern dynamics of our system. In the present paper, we will focus on the stability of the uniform oscillation $A_{0}(t)$ for which

$$
\begin{gathered}
R=1, \\
\Omega_{0}=-c_{2} .
\end{gathered}
$$

We now put

$$
A(\boldsymbol{r}, t)=[1+\Delta(\boldsymbol{r}, t)] A_{0}(t),
$$

and linearize Eq. (30) in $\Delta(\boldsymbol{r}, t)$. The linearized equation can be solved in terms of the Fourier components of $\Delta(\boldsymbol{r}, t)$, denoted by $\Delta_{q}(t)$. Assuming its time dependence in the form $\Delta_{q}(t) \propto \exp (\lambda t)$, we find the eigenvalue equation

$$
\lambda^{2}+u(q) \lambda+v(q)=0
$$

where

$$
\begin{gathered}
u(q)=-2 \operatorname{Re} \gamma(q), \\
v(q)=|\gamma(q)|^{2}-|\gamma(0)|^{2},
\end{gathered}
$$

with

$$
\gamma(q)=-\left(1+i c_{2}\right)-\left(\delta_{1}+i \delta_{2}\right) q^{2}+K\left(1+i c_{1}\right)\left(G_{q}-G_{0}\right) .
$$

In what follows, we shall first concentrate on the case without diffusive coupling, i.e., $\delta_{1}=\delta_{2}=0$; the effects of nonvanishing but small $\delta_{1}$ will be touched upon later.

Let the solutions of Eq. (39) be denoted by $\lambda_{+}$and $\lambda_{-}$ with $\operatorname{Re} \lambda_{+} \geqslant \operatorname{Re} \lambda_{-}$. The uniform oscillation is stable if and only if $\operatorname{Re} \lambda_{+}<0$ holds for all $q$. This holds only when $u(q)>0$ and $v(q)>0$ for all $q$. Equivalently, if one of these inequalities becomes violated for a certain $q$, then the uniform oscillation loses stability. This implies that the types of instability could be classified in terms of the signs of $u(q)$ and $v(q)$. Simple calculation shows that $u(q)$ and $v(q)$ can be expressed in the following form, where we use the notation $Q \equiv q^{2}$.

$$
\begin{gathered}
u(q)=\xi(Q) \xi_{0}(Q), \\
v(q)=\zeta(Q) \zeta_{0}(Q) Q .
\end{gathered}
$$

Here $\xi_{0}(Q)$ and $\zeta_{0}(Q)$ are non-negative functions of $Q$, and

$$
\begin{gathered}
\xi(Q) \equiv a_{2} Q^{2}+a_{1} Q+a_{0}, \\
\zeta(Q) \equiv b_{1} Q+b_{0}, \\
a_{0}=1+\theta^{2},
\end{gathered}
$$




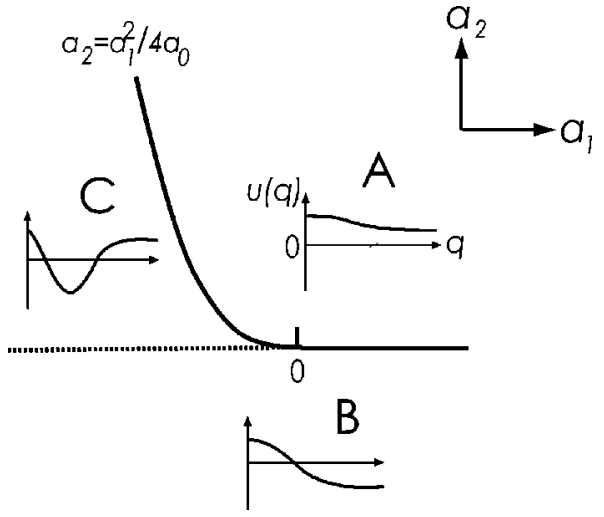

FIG. 1. The sign of $u(q)$ changes with $q$ in three different ways. The figure shows how the corresponding domains $A, B$, and $C$ appear in the $a_{1}-a_{2}$ plane. $u(q)$ is positive for all $q$ in $A$, negative above some critical $q$ in $B$, and negative in a finite interval of $q$ in $C$. The line separating domains $A$ and $C$ is given by a parabola $a_{2}$ $=a_{1}^{2} / 4 a_{0}$.

$$
\begin{gathered}
a_{1}=2+K\left(1+c_{1} \theta\right), \\
a_{2}=1+K, \\
b_{0}=2 K\left[1+c_{1} c_{2}+\left(c_{1}-c_{2}\right) \theta\right], \\
b_{1}=K\left[2\left(1+c_{1} c_{2}\right)+K\left(1+c_{1}^{2}\right)\right] .
\end{gathered}
$$

Figure 1 shows the $a_{1}-a_{2}$ plane divided into three domains (labeled by $A, B$, and $C$ ) corresponding to qualitatively different forms of $u(q)$. Note that the $a_{1}-a_{2}$ plane covers all possible types of $u(q)$ because $a_{0}$ is positive definite. Similar picture for $v(q)$ in the $b_{0}-b_{1}$ plane is displayed in Fig. 2 where the whole space is divided into four domains (labeled by $A^{\prime}$ to $D^{\prime}$ ). Since the parameters $a_{1}, a_{2}, b_{0}$, and $b_{1}$ as functions of four independent parameters $K, \theta, c_{1}$, and $c_{2}$

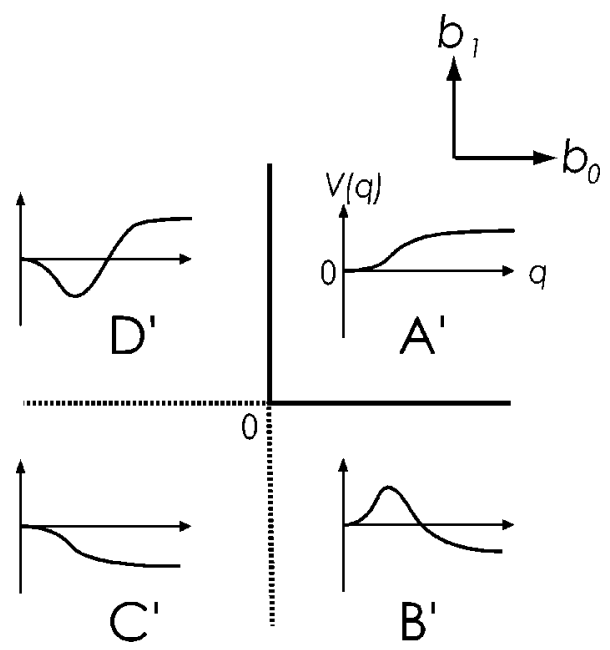

FIG. 2. The sign of $v(q)$ changes with $q$ in four different ways. The figure shows how the corresponding domains $A^{\prime}, B^{\prime}, C^{\prime}$, and $D^{\prime}$ appear in the $b_{0}-b_{1}$ plane. $v(q)$ is positive for all $q$ in $A^{\prime}$, negative above some critical $q$ in $B^{\prime}$, negative for all $q$ in $C^{\prime}$, and negative below some critical $q$ in $D^{\prime}$.

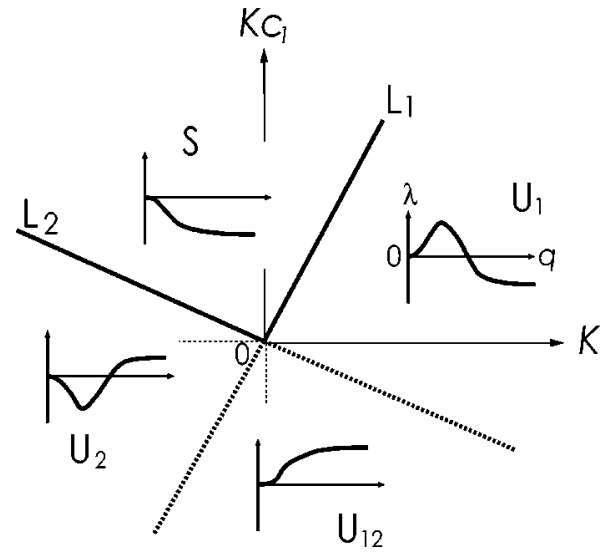

FIG. 3. Four types of eigenvalue spectrum for the phaselike fluctuations about the uniform oscillation which is stable only for type $S$. The figure shows how they appear in the $K-K c_{1}$ plane in the vicinity of the origin $K=0$. The other parameters are fixed as $c_{2}$ $=1.0$ and $\theta=6.0$. In each of the four cases, the eigenvalues of the amplitudelike fluctuations, which is not shown in the figure, form a branch completely separated from the phase branch and remain negative for all $q$.

can be changed independently, every combination between two members, one from the group $(A, B, C)$ and the other from $\left(A^{\prime}, B^{\prime}, C^{\prime}, D^{\prime}\right)$, is possible. Uniform oscillation is unstable for all these combinations except for $\left(A, A^{\prime}\right)$. Loss of stability occurs as we move across the line $a_{2}=0$ or $a_{1}^{2}$ $-4 a_{0} a_{2}=0$ in Fig. 1, for which the instability is oscillatory, or otherwise $b_{0}=0$ or $b_{1}=0$ in Fig. 2, for which the instability is nonoscillatory. The critical wave number for which $\operatorname{Re} \lambda<0$ becomes first violated equals zero on the line $b_{0}$ $=0$, finite on $a_{1}^{2}-4 a_{0} a_{2}=0$, and infinite on $a_{2}=0$ and $b_{1}$ $=0$. The last type of instability, i.e., the instability which starts at infinite $q$, was called short-wavelength bifurcation by Heagy et al. [7].

If $|K|$ is not too large, $u$ is non-negative, so that the instability is only through $v$ becoming negative and hence it is nonoscillatory. The corresponding critical lines $b_{0}=0$ and $b_{1}=0$ in Fig. 2 can now be translated into critical relations between $K$ and $K c_{1}$ under fixed $c_{2}$ and $\theta$. In this way, we have four types of eigenvalue spectrum shown schematically in Fig. 3 where lines $L_{1}$ and $L_{2}$ correspond to $b_{0}=0$ and $b_{1}=0$, respectively. The figure shows the spectra only for phaselike fluctuations because in each case the amplitude branch which is separated from the phase branch remains negative for all $q$ so that the amplitudelike fluctuations are irrelevant to stability. Separation between the phase and amplitude branches also implies that the eigenvalues associated with these branches are all real. In contrast, if these branches merge, then the eigenvalues of the two branches for given $q$ would form a complex conjugate pair. Note that in each type of spectrum shown in the figure, the eigenvalue saturates to a constant as the wave number tends to infinity, which is characteristic to nonlocally coupled systems. The characteristic wave number about which the eigenvalue starts to saturate equals the inverse of the coupling radius. Physically, this reflects the fact that the dynamics of fluctuations whose wavelengths are much shorter than the coupling radius is 


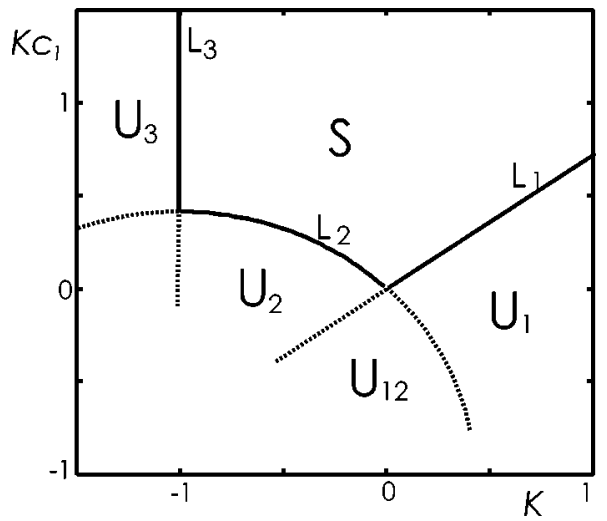

FIG. 4. Figure 3 is extended to the region of larger $K$. A new type of eigenvalue spectrum appears for large negative $K$. This type, labeled by $U_{3}$ is similar to $U_{2}$ in Fig. 3 except that the amplitude branch merges with the phase branch above a certain wave number which is still below the wave number at which $\operatorname{Re} \lambda$ changes sign. Thus, the eigenvalues associated with the unstable fluctuations are complex rather than real, i.e., the instability is oscillatory in nature.

practically the same as the oscillators' individual dynamics, and hence it is insensitive to the wave number. Note also that the type $U_{2}$ spectrum is possible only when $\theta$ is nonvanishing or, equivalently, when the nonlocal coupling before reduction involves delay.

For larger $|K|$, the phase and amplitude branches can merge, so that oscillatory instability becomes possible. In particular, the short-wavelength-type instability, i.e., the instability initiated by fluctuations with infinite wave number can occur across the line $a_{2}=0$ or $K=-1$. This type actually appears in the stability diagram in Fig. 4 which is a global extension of the diagram in Fig. 3. The values of the other parameters are the same as in Fig. 3, i.e., $c_{2}=1.0$ and $\theta=6.0$. Oscillatory instability initiated by fluctuations with finite wave number, which occurs when crossing the line $a_{1}^{2}-4 a_{0} a_{2}=0$ in Fig. 1, does not appear in Fig. 4, but may appear when different values of $c_{2}$ and $\theta$ are chosen. For instance, the stability diagram for the case of $c_{2}=-2.5$ and $\theta=1.5$ is displayed in Fig. 5 where the line $L_{4}$ gives the

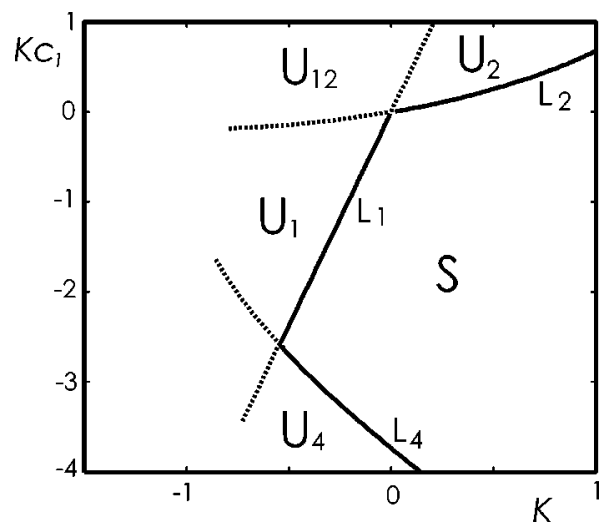

FIG. 5. Similar to Fig. 4 but for different values of $c_{2}$ and $\theta$, i.e., $c_{2}=-2.5$ and $\theta=1.5$. New type of eigenvalue spectrum, denoted by $U_{4}$, appears which differs from $U_{3}$ in Fig. 4 only in that the eigenvalues saturate to a negative value as $q$ goes to infinity.

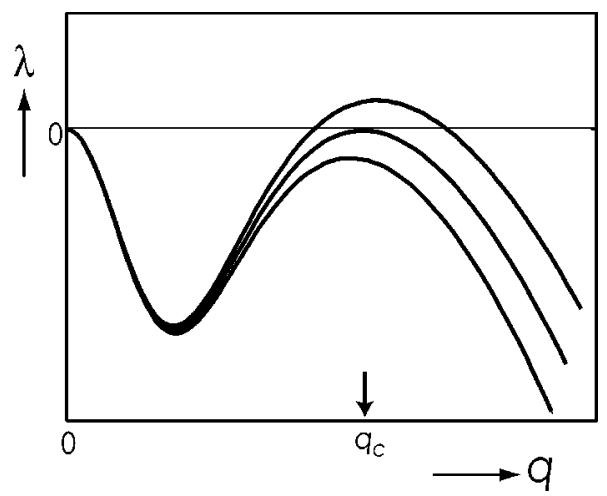

FIG. 6. Eigenvalue spectra close to a finite-wave-number instability, which are obtained as a modification of $U_{2}$ in Fig. 3 by assuming nonvanishing $\delta_{1}$ in the reduced equation (30).

boundary associated with the last type of instability.

Finally, we comment on the effects of weak diffusion represented by the term $\left(\delta_{1}+i \delta_{2}\right) \nabla^{2} A$ in Eq. (30). For simplicity, we assume that $\delta_{2}$ is vanishing while $\delta_{1}$ is a small positive. It is clear that this diffusion term simply gives rise to an additional stabilizing term $-\delta_{1} q^{2}$ to each of the eigenvalues $\lambda_{ \pm}$as a function of $q$. Thus, the fluctuations with sufficiently short wavelength are always decaying. A particularly interesting result from this fact appears in type $U_{2}$ eigenvalue spectrum in Fig. 3. Depending on the value of $\delta_{1}$, the dispersion curve is deformed like some curves shown in Fig. 6. Even the dispersion curves of types $U_{1}$ and $U_{12}$ could be deformed into a similar form if some parameter vaules are chosen suitably. It is clear that there is a critical value of $\delta_{1}$ at which fluctuations with a certain wave number $q_{c}$ become unstable. Due to the presence of long-wavelength fluctuations which are almost neutral in stability, the unstable growth of the mode with $q_{c}$ will generally be unable to lead to a Turing-type periodic standing pattern. Instead, from the outset, a group of modes with wave numbers close to $q_{c}$ will couple nonlinearly with another group of modes of almost vanishing $q$ with almost neutral stability, leading to a peculiar spatiotemporal chaos $[8,13]$. The simple evolution equation,

$$
\partial_{t} u=-\partial_{x}^{2}\left[\epsilon-\left(1+\partial_{x}^{2}\right)^{2}\right] u-\left(\partial_{x} u\right)^{2},
$$

called the Nikolaevskii equation [10], gives qualitatively the same eigenvalue spectrum as those in Fig. 6. Actually, it was argued previously that the Turing pattern in this system existing for small positive $\epsilon$ is always unstable, and as a result the system immediately becomes turbulent characterized by the coexistence of turbulent fluctuations with vastly different length scales. Possibility of this type of turbulence in reaction-diffusion systems was discussed by Fujisaka and Yamada [11]. In electroconvective systems, similar type of complex behavior was discovered by Kai et al. [9] which they called soft-mode turbulence.

\section{SUMMARY}

If spatially distributed limit-cycle oscillators are diffusively coupled not directly but via a certain diffusive com- 
ponent, the oscillators may be viewed as coupled nonlocally after a mathematical elimination of this diffusive variable. We showed in the present paper that this actually occurs in reaction-diffusion systems and that the nonlocality of this kind persists even close to the Hopf bifurcation point provided the coupling between each local oscillator and the diffusive component is sufficiently weak. Specifically, under this condition, the system is reduced to a complex GinzburgLandau-type equation with nonlocal rather than diffusive coupling. Temporal nonlocality or memory effects which generally exist before reduction does not appear explicitly in the reduced equation, still they may generally affect the functional form of the coupling function. Our results were generalized so as to include the effects of direct but weak diffu- sive coupling among the oscillators.

Linear stability analysis of the uniformly oscillating state of the reduced equation was also carried out. Some new types of eigenvalues spectrum were found to arise, and their physical relevance was suggested.

How the solution behaves in the nonlinear regime is known only partially. For instance, type $U_{12}$ spectrum in Fig. 3 was found to lead to turbulence with multiaffinity [5]. It was also found that even the normal spectrum (type $S$ ) can give rise to peculiar spiral waves without phase singularity when the nonlocal coupling becomes weak [12]. Different aspects of the nonlocal CGL including the aforementioned type of turbulence associated with the dispersion curve in Fig. 6 will be developed in forthcoming papers [13].
[1] Y. Kuramoto, Chemical Oscillation, Waves, and Turbulence (Springer, New York, 1984).

[2] M.C. Cross and P.C. Hohenberg, Rev. Mod. Phys. 65, 851 (1993).

[3] T. Bohr et al., Dynamical Systems Approach to Turbulence (Cambridge University Press, Cambridge, 1998).

[4] Y. Kuramoto, Prog. Theor. Phys. 94, 321 (1995).

[5] Y. Kuramoto and H. Nakao, Physica D 103, 294 (1997); Y. Kuramoto, D. Battogtokh, and H. Nakao, Phys. Rev. Lett. 81, 3543 (1998); Y. Kuramoto, H. Nakao, and D. Battogtokh, Physica A 288, 244 (2000).

[6] V.K. Vanag and I.R. Epstein, Phys. Rev. Lett. 87, 228301 (2001).

[7] J.F. Heagy, L.M. Pecora, and T.L. Carroll, Phys. Rev. Lett. 74, 4185 (1995).
[8] M.I. Tribelsky and M.G. Velarde, Phys. Rev. E 54, 4973 (1996); M.I. Tribel'skii, Phys. Usp. 40, 159 (1997); M.I. Tribelsky and K. Tsuboi, Phys. Rev. Lett. 76, 1631 (1996).

[9] S. Kai, K. Hayashi, and Y. Hidaka, J. Phys. Chem. 100, 19007 (1996).

[10] V.N. Nikolaevskii, in Recent Advances in Engineering Science, edited by S.L. Koh and C.G. Speciale, Lecture Notes in Engineering Vol. 39 (Springer-Verlag, Berlin, 1989), p. 210.

[11] H. Fujisaka and T. Yamada, Prog. Theor. Phys. 106, 315 (2001).

[12] Y. Kuramoto and S. Shima, Prog. Theor. Phys. Suppl. (to be published), nlin.PS/0304021.

[13] Similar complex dynamics arising in our three-component reaction-diffusion model and also in nonlocal CGL is being studied by D. Tanaka (unpublished). 